University of South Florida

DIGITAL COMMONS

Digital Commons @ University of

@ UNIVERSITY OF SOUTH FLORIDA

South Florida

USF Accountability Reports

USF Archives

$1-1-2020$

\title{
2020 Accountability Plan USF
}

USF

Follow this and additional works at: https://digitalcommons.usf.edu/usf_accountability_reports

\section{Scholar Commons Citation}

USF, "2020 Accountability Plan USF" (2020). USF Accountability Reports. 77.

https://digitalcommons.usf.edu/usf_accountability_reports/77

This Article is brought to you for free and open access by the USF Archives at Digital Commons @ University of South Florida. It has been accepted for inclusion in USF Accountability Reports by an authorized administrator of Digital Commons @ University of South Florida. For more information, please contact digitalcommons@usf.edu. 
2020

\section{ACCOUNTABILITY PLAN}

\section{UNIVERSITY OF \\ SOUTH FLORIDA}

USF BOT Approved, 4/16/2020

Revised by Board of Governors Staff, 7/9/2020

The following sections were approved by the Board Governors on May 28, 2020 \& July 21, 2020: academic program coordination, graduation rate improvement plan update, $\&$ the historical data reported for the performance-based funding metrics, key performance indicators, enrollment planning, \& preeminent research university funding metrics. 


\section{INTRODUCTION}

The Accountability Plan is an annual report that is closely aligned with the Board of Governors' 2025 System Strategic Plan. This report enhances the System's commitment to accountability and strategic planning by fostering greater coordination between institutional administrators, University Boards of Trustees and the Board of Governors regarding each institution's direction and priorities as well as performance expectations and outcomes on institutional and System-wide goals.

Once an Accountability Plan is approved by each institution's respective Boards of Trustees, the Board of Governors will review and consider the plan for approval, excluding those sections of the Plan that require additional regulatory or procedural approval pursuant to law or Board regulations. 


\section{STRATEGY}

\section{Mission Statement}

Led by outstanding faculty and professional staff, the University of South Florida (USF) conducts innovative scholarship, creative activity, and basic and translational research, and delivers a world-class educational experience promoting the success of our talented and diverse undergraduate, graduate, and professional students.

As a public metropolitan research university, USF, in partnership with our communities, serves the people of Florida, the nation, and the world by fostering intellectual inquiry and outcomes that positively shape the future regionally, nationally, and globally.

\section{Statement of Strategy}

The University of South Florida, comprised of campuses in Tampa, St. Petersburg, and Sarasota-Manatee, is guided by the Florida Board of Governors' (BOG) Strategic Plan and driven by a commitment to accountability and excellence. USF is committed to "Students First" by expanding access and raising educational attainment while continuing to strengthen USF's stature as a Preeminent Research University and its national and global rankings. USF continues national best practices in student success and diversity while maintaining the highest levels of academic excellence and high-impact research.

The 2018 Florida Legislature mandated that by July 1, 2020, USF will consolidate into one university, geographically distributed. "One USF" affords the State of Florida the opportunity to maximize the impact of a highly-ranked research university located across a large metropolitan area. USF is poised to continue its upward trajectory as the nation's fastest-rising university under our plan for "One USF". Central to our consolidation strategy is leveraging the distinct advantages of each USF campus while aligning accountability across campuses, valuing shared governance through engaging students, faculty and staff. Contingent upon legislative support, major investments in all three campuses will raise the national profile of "One USF" and provide students and faculty with new academic and research opportunities.

President Steven C. Currall initiated a Strategic Renewal process during the Fall of 2019, and an early step of the process is the establishment of USF's Principles of Community Taskforce. The process is designed to strengthen a campus climate of mutual support among faculty, staff, and students and will facilitate our shared strategy, mission, vision, and goals for "One USF". 


\section{STRATEGY (cont.)}

\section{Strengths, Opportunities \& Challenges}

USF is on an unprecedented trajectory in reputation and performance metrics, gaining national recognition as one of the fastest rising universities. We continue to make gains by increasing student success outcomes, improving FTIC retention rates and four-year and six-year graduation rates, and lowering student-faculty ratio. USF looks forward to implementing a strategic renewal plan, which will inform our academic and research priorities. USF is uniquely positioned to train the next generation of physicians and pioneer world-changing discoveries at the new state-of-the-art USF Health Morsani College of Medicine and Taneja College of Pharmacy and Heart Institute which opened in January 2020.

As we continue on our path as "One USF", we will utilize best practices: increasing faculty resources and productivity, enhancing the quality of access and programs for incoming students, cultivating institutional reputation among peer intuitions, and growing community support through philanthropy, while aligning with the Florida Legislature's vision. We will continue our upward trajectory, uphold Preeminence, and maintain our status as a top global university. Major investments in all three campuses, contingent upon legislative support, will raise the national profile of "One USF" and provide students and faculty with new academic and research opportunities. This support is fundamental in offering new scholarly opportunities for faculty and students at each campus. The next step in the consolidation process is the SACSCOC site visit following July 1, 2020 to affirm we are working as one university. USF faculty and staff across all three campuses continue to work diligently to complete all SACSCOC substantive tasks and prepare documentation, assuring we have a smooth transition as we work towards "One USF"

\section{Three Key Initiatives \& Investments}

USF is an institution where excellence and opportunity converge. We focus on the quality of undergraduate and graduate students and their educational experience; faculty, research and creative impact; and strategic partnerships. Supporting initiatives include 1. Academic and Research Excellence, 2. Accreditation for Consolidation, and 3. Strengthening Preeminence with a continued path towards AAU membership eligibility and becoming Top 25 in USNWR's ranking. Across all of our campuses, we will increase our efforts in:

Advancing student learning, success, persistence, retention, and degree completion through the Excellence in Academic Advising initiative; continually enhancing and refining the MWell4Success program which includes an array of support services from therapy to time-management, anxiety-reducing skills, and stress coping strategies; advancing at least one center of excellence on each USF campus under "One USF"; launching the Faculty Success Workgroup to focus on matters of significant importance in strengthening the Preeminent stature of a consolidated USF, such as helping to increase the research portfolio with strategic hires and driving down student to faculty ratio; and continuing a General Education Council to advise on the ongoing development, implementation and assessment of an effective general education program and expanded course offerings across all three campuses. 


\section{ACCOUNTABILITY PLAN}

University of South Florida

USF BOT Approved, 4/16/2020

\section{STRATEGY (cont.) \\ Graduation Rate Improvement Plan Update}

The Graduation Rate Improvement plan implemented by the USF System in 2018 built on and enhanced comprehensive, system-wide initiatives then underway, including, but not limited to 1) predictive analytics, 2) case management, 3) course scheduling, and 4) the Finish in Four program. USF has made substantial progress in each one of these areas.

1). Predictive Analytics: The Civitas Learning analytics platform has been extended to users at USFSP and USFSM. Lists of at-risk students are distributed around the campuses every week. Further, our institutional research office worked with Civitas to modify the platform to predict a students' likelihood of persistence to the following fall semester (rather than semester to semester). In addition, the in-house predictive models built on pre-enrollment data and surveys (known as the First Year Retention model), were also developed for USFSP and USFSM.

2). Case Management: The cross-functional Persistence Committees have been strengthened at all three campuses by standardizing the practices utilized by the professional staff. In addition, Information Technology enhanced the case management communications platform, Archivum Insights, to allow for appointment scheduling and text messaging. Support personnel across the three campuses are developing and operating under a common "playbook" to share best practices and train additional personnel.

3) Course Scheduling: Enrollment planners and college schedulers promoted the utilization of the Ad Astra platform to assist in developing course schedules that optimize seats and facilitate timely degree completion. Through training sessions and regular meetings, we promoted the concept and practice of scheduling courses that serve students' needs and demands first. We also launched curricular "audits" of all degree programs to identify bottlenecks and other obstacles built into the curriculum that delay a students' path to a degree.

4) Finish in Four: To encourage students to graduate in four years, the university awarded $\$ 298,555$ in financial incentives to FTIC students in the 2015 cohorts who were just short of 120 credit hours. The awards contributed to an increase in the USF Tampa four-year graduation rate from 55\% in 2016 to $61.5 \%$ in 2019 . In 2017, USF developed the Green to Gold Grant program to offer additional financial incentives to Florida resident summer/fall FTIC admits who have a zero expected family contribution. This grant, in combination with the Federal Pell Grant, covers tuition, fees and books. To date, we have paid 535 students $\$ 1,185,209$ for $2019-20$. Bright Futures recipients were also eligible for this grant. 


\section{Key Achievements for Last Year (Student, Faculty, Program, Institutional)}

\section{Student}

1. Two USF Judy Genshaft Honors College students, Keller Blackwell and Willie McClinton, were named Goldwater Scholars for 2019 , chosen out of nearly 1,300 students nationally. The highly competitive scholarship is the most prestigious undergraduate award in the country for science research and provides $\$ 7,500$ for educational expenses and research support.

2. A student-led initiative at USF St. Petersburg led to the installation in the student dining facility of an ORCA Biodigester, which digests food waste using microorganisms that thrive in cold water. Less than six months after it was installed, the ORCA has diverted more than 3,500 pounds of food waste from landfills.

3. The USF Sarasota-Manatee campus hosted a "Reverse Career Fair" that changed the format of a traditional career fair by providing students with an opportunity to set up tables to showcase their skills and experiences to recruiters in attendance. The unique format was recognized by the National Association of Colleges and Employers (NACE) as a national best practice. In a post-event survey, 17 of 22 students who responded reported that they were offered at least one job and/or internship; 14 students accepted positions.

Faculty

1. The Chronicle of Higher Education ranked USF \#1 in the nation for Top Producer of Fulbright Scholars, with 12 faculty scholars for the 2018-2019 academic year.

2. USF ranked 4th worldwide among organizations with the most Fellows of the American Association for the Advancement of Science (AAAS) elected in 2019, a standing USF has held for the past five years. As of 2019, USF has a total of 73 AAAS Fellows among its faculty.

3. USF faculty continue to earn national and international recognition. In 2019, USF faculty received 138 new external honors and awards.

\section{Program}

1. USF St. Petersburg launched the Innovation Scholars program, which matches first-year students with mentors from local businesses for job-shadowing opportunities. Thirty-three students were paired with 29 different businesses from St. Petersburg in Fall 2019, and another 28 were paired for spring 2020. Intent is to double that number next year.

2. The Morsani College of Medicine Class of 2023 is one of the most competitive in the college's history. With more than 5,500 applications, students had less than a 4 percent chance of getting in. This class attained an average MCAT score of 515, putting them in the 93rd percentile nationally with an average GPA of 3.76 (2019).

3. The USF Sarasota-Manatee campus was awarded the CareerSource Suncoast Workforce Education Partner of the Year for our innovative work in connecting students to employers for internships and employment upon graduation.

\section{Institutional}

1. The University of South Florida was a recipient of the 2019 American Council on Education/Fidelity Investments Award for Institutional Transformation. The award was created to recognize institutions that have responded to higher education challenges in innovative and creative ways and achieved dramatic changes in a relatively brief period. It includes a $\$ 10,000$ prize.

2. Helios Education Foundation has awarded the University of South Florida's Black Leadership Network (BLN) a \$2.1 million grant to support the future success of underrepresented students across USF. USF won the Association of Public and Land-grant Universities' (APLU) 2019 Institutional Award for Global Learning, Research \& Engagement. The Platinum Level APLU award focuses on four areas of excellence: inclusivity, internationalization of research and engagement, leadership and pervasiveness, and assessment. 


\section{PERFORMANCE-BASED FUNDING METRICS}

\section{Percent of Bachelor's Graduates Enrolled or Employed (\$25,000+)}

\begin{tabular}{cccccccccccc} 
& $2013-14$ & $2014-15$ & $2015-16$ & $2016-17$ & $2017-18$ & $2018-19$ & $2019-20$ & $2020-21$ & $2021-22$ & $2022-23$ \\
\hline ACTUAL & 67.2 & 69.6 & 70.0 & 70.4 & 71.6 &. &. &. &. &. \\
APPROVED GOALS &. & 66.8 & 70.5 & 70.5 & 71.4 & 72.5 & 73.1 & 74 &. &. \\
PROPOSED GOALS &. &. &. &. &. & 72.8 & 73.1 & 74.0 & 74.5 & 75.0
\end{tabular}

\section{Median Wages of Bachelor's Graduates Employed Full-time}

\begin{tabular}{ccccccccccc} 
& $2013-14$ & $2014-15$ & $2015-16$ & $2016-17$ & $2017-18$ & $2018-19$ & $2019-20$ & $2020-21$ & $2021-22$ & $2022-23$ \\
\hline ACTUAL & 36,700 & 38,000 & 37,300 & 38,000 & 39,400 &. &. &. &. &. \\
APPROVED GOALS &. & 36,300 & 38,600 & 39,100 & 38,768 & 40,375 & 40,980 & 41,544 &. &. \\
PROPOSED GOALS &. &. &. &. &. & 40,375 & 40,980 & 41,544 & 42,000 & 42,500
\end{tabular}

3. Average Cost to the Student [Net Tuition \& Fees per 120 Credit Hours for Resident Undergraduates]

\begin{tabular}{ccccccccccc} 
& $2014-15$ & $2015-16$ & $2016-17$ & $2017-18$ & $2018-19$ & $2019-20$ & $2020-21$ & $2021-22$ & $2022-23$ & $2023-24$ \\
\hline ACTUAL & 13,540 & 13,280 & 12,040 & 7,130 & 4,330 &. &. &. &. &. \\
APPROVED GOALS &. &. & 13,000 & 12,900 & 7,110 & 7,110 & 7,110 & 7,110 &. &. \\
PROPOSED GOALS &. &. &. &. &. & 7,110 & 7,110 & 7,110 & 7,110 & 7,110
\end{tabular}

4. FTIC Four-Year Graduation Rate [Full-time FTIC students]

\begin{tabular}{ccccccccccc} 
& $2011-15$ & $2012-16$ & $2013-17$ & $2014-18$ & $2015-19$ & $2016-20$ & $2017-21$ & $2018-22$ & $2019-23$ & $2020-24$ \\
\hline ACTUAL & 48.8 & 52.0 & 57.4 & 58.6 & 59.4 &. &. &. &. &. \\
APPROVED GOALS &. & 50.4 & 53 & 57.6 & 59.2 & 61.8 & 64.1 &. &. &. \\
PROPOSED GOALS &. &. &. &. &. & 61.8 & 64.1 & 64.5 & 65.0 & 65.5
\end{tabular}

5. Academic Progress Rate [Second Fall Retention Rate with at Least a 2.0 GPA for Full-time FTIC students]

\begin{tabular}{ccccccccccc} 
& $2014-15$ & $2015-16$ & $2016-17$ & $2017-18$ & $2018-19$ & $2019-20$ & $2020-21$ & $2021-22$ & $2022-23$ & $2023-24$ \\
\hline ACTUAL & 85.1 & 86.1 & 86.2 & 86.6 & 87.7 &. &. &. &. &. \\
APPROVED GOALS &. & 85.6 & 87.5 & 87.4 & 89.0 & 90.3 & 90.8 & 91.4 &. &. \\
PROPOSED GOALS &. &. &. &. &. & 90.3 & 90.8 & 91.4 & 91.5 & 91.6
\end{tabular}




\section{PERFORMANCE-BASED FUNDING METRICS (cont.)}

\section{Percentage of Bachelor's Degrees Awarded within Programs of Strategic Emphasis}

\begin{tabular}{ccccccccccc} 
& $2014-15$ & $2015-16$ & $2016-17$ & $2017-18$ & $2018-19$ & $2019-20$ & $2020-21$ & $2021-22$ & $2022-23$ & $2023-24$ \\
\hline ACTUAL & 54.6 & 59.0 & 61.4 & 61.5 & 62.8 &. &. &. &. &. \\
APPROVED GOALS &. & 54.8 & 59.2 & 61.7 & 62.2 & 63.5 & 64.5 & 65.4 &. &. \\
PROPOSED GOALS &. &. &. &. &. & 63.5 & 64.5 & 65.4 & 65.5 & 65.6
\end{tabular}

7. University Access Rate [Percent of Undergraduates with a Pell grant]

\begin{tabular}{ccccccccccc} 
& FALL & FALL & FALL & FALL & FALL & FALL & FALL & FALL & FALL & FALL \\
& 2014 & 2015 & 2016 & 2017 & 2018 & 2019 & 2020 & 2021 & 2022 & 2023 \\
\hline ACTUAL & 43.0 & 41.2 & 40.0 & 41.7 & 40.0 &. &. &. &. &. \\
APPROVED GOALS &. & 40 & 41 & 40.5 & 42.2 & 42.2 & 42.2 & 42.2 &. &. \\
PROPOSED GOALS &. &. &. &. &. & 42.2 & 42.2 & 42.2 & 42.2 & 42.2
\end{tabular}

8. Percentage of Graduate Degrees Awarded within Programs of Strategic Emphasis

\begin{tabular}{ccccccccccc} 
& $2014-15$ & $2015-16$ & $2016-17$ & $2017-18$ & $2018-19$ & $2019-20$ & $2020-21$ & $2021-22$ & $2022-23$ & $2023-24$ \\
\hline ACTUAL & 72.7 & 74.6 & 74.8 & 73.3 & 72.2 &. &. &. &. &. \\
APPROVED GOALS &. & 74 & 74.1 & 75.2 & 73.6 & 73.8 & 73.8 & 73.8 &. &. \\
PROPOSED GOALS &. &. &. &. &. & 73.8 & 73.8 & 73.8 & 74.5 & 75.0
\end{tabular}

\section{BOG Choice: Percent of Baccalaureate Degrees Awarded Without Excess Hours}

\begin{tabular}{ccccccccccc} 
& $2014-15$ & $2015-16$ & $2016-17$ & $2017-18$ & $2018-19$ & $2019-20$ & $2020-21$ & $2021-22$ & $2022-23$ & $2023-24$ \\
\hline ACTUAL & 65.8 & 75.6 & 78.3 & 80.7 & 84.7 &. &. &. &. &. \\
APPROVED GOALS &. & 68.1 & 77.5 & 79.0 & 81.6 & 81.7 & 82.6 & 83.1 &. &. \\
PROPOSED GOALS &. &. &. &. &. & 85.0 & 85.2 & 85.4 & 85.6 & 85.8
\end{tabular}

10. BOT Choice: 6-Year Graduation Rates [Full- \& Part-time students]

\begin{tabular}{cccccccccccc} 
& $2009-15$ & $2010-16$ & $2011-17$ & $2012-18$ & $2013-19$ & $2014-20$ & $2015-21$ & $2016-22$ & $2017-23$ & $2018-24$ \\
\hline ACTUAL & 67.7 & 66.4 & 68.8 & 71.2 & 73.2 &. &. &. &. &. \\
APPROVED GOALS &. & 66.7 & 70 & 69 & 71.3 & 72.1 & 72.1 & 73.3 &. &. \\
PROPOSED GOALS &. &. &. &. &. & 74.0 & 74.5 & 75.0 & 75.5 & 76.0
\end{tabular}




\section{PREEMINENT RESEARCH UNIVERSITY FUNDING METRICS}

*USF-TAMPA ONLY*

A. (1). Average GPA

\begin{tabular}{ccccccccccc} 
& FALL & FALL & FALL & FALL & FALL & FALL & FALL & FALL & FALL & FALL \\
& 2015 & 2016 & 2017 & 2018 & 2019 & 2020 & 2021 & 2022 & 2023 & 2024 \\
\hline ACTUAL & 4.1 & 4.1 & 4.1 & 4.1 & 4.1 &. &. &. &. &. \\
APPROVED GOALS &. & 4.0 & 4.1 & 4.1 & 4.1 & 4.1 & 4.1 & 4.1 &. &. \\
PROPOSED GOALS &. &. &. &. &. & 4.2 & 4.2 & 4.2 & 4.2 & 4.2
\end{tabular}

A. (2). Average SAT Score

\begin{tabular}{ccccccccccc} 
& FALL & FALL & FALL & FALL & FALL & FALL & FALL & FALL & FALL & FALL \\
& 2015 & 2016 & 2017 & 2018 & 2019 & 2020 & 2021 & 2022 & 2023 & 2024 \\
\hline ACTUAL & $1223^{*}$ & $1226^{*}$ & 1280 & 1296 & 1287 &. &. &. &. &. \\
APPROVED GOALS &. & 1220 & 1280 & 1282 & 1296 & 1300 & 1302 & 1305 &. &. \\
PROPOSED GOALS &. &. &. &. &. & 1312 & 1315 & 1318 & 1321 & 1324
\end{tabular}

Note: Historical scores/goals were based on a different SAT scale standard.

B. Public University National Ranking [Top50 rankings based on BOG's official list of publications]

\begin{tabular}{cccccccccccc} 
& 2016 & 2017 & 2018 & 2019 & 2020 & 2021 & 2022 & 2023 & 2024 & 2025 \\
\hline ACTUAL & 4 & 4 & 4 & 4 & 6 &. &. &. &. &. \\
APPROVED GOALS &. & 3 & 5 & 5 & 5 & 5 & 5 & 5 &. &. \\
PROPOSED GOALS &. &. &. &. &. & 6 & 6 & 6 & 7 & 7
\end{tabular}

C. Freshman Retention Rate [Full-time FTIC students]

\begin{tabular}{cccccccccccc} 
& $2014-15$ & $2015-16$ & $2016-17$ & $2017-18$ & $2018-19$ & $2019-20$ & $2020-21$ & $2021-22$ & $2022-23$ & $2023-24$ \\
\hline ACTUAL & 88 & 90 & 90 & 91 & 91 &. &. &. &. &. \\
APPROVED GOALS &. & 90 & 91 & 91 & 91 & 92 & 93 & 93 &. &. \\
PROPOSED GOALS &. &. &. &. &. & 92 & 93 & 93 & 93 & 93
\end{tabular}

D. Four-year Graduation Rate [Full-time FTIC students only]

\begin{tabular}{ccccccccccc} 
& $2011-15$ & $2012-16$ & $2013-17^{*}$ & $2014-18$ & $2015-19$ & $2016-20$ & $2017-21$ & $2018-22$ & $2019-23$ & $2020-24$ \\
\hline ACTUAL & 51 & 55 & 60 & 61 & 62 &. &. &. &. &. \\
APPROVED GOALS &. & 50 & 56 & 60 & 62 & 64 & 65 & 68 &. &. \\
PROPOSED GOALS &. &. &. &. &. & 64 & 65 & 68 & 68 & 68
\end{tabular}

Note*: The 2013-17 data will be reported to IPEDS in 2020 as part of their annual data collection cycle. 


\section{PREEMINENT RESEARCH UNIVERSITY FUNDING METRICS (cont.)}

\section{E. National Academy Memberships}

\begin{tabular}{ccccccccccc} 
& 2016 & 2017 & 2018 & 2019 & 2020 & 2021 & 2022 & 2023 & 2024 & 2025 \\
\hline ACTUAL & 8 & 11 & 13 & 14 & 15 &. &. &. &. &. \\
APPROVED GOALS &. & 9 & 10 & 13 & 14 & 15 & 16 & 17 &. &. \\
PROPOSED GOALS &. &. &. &. &. & 15 & 16 & 17 & 17 & 17
\end{tabular}

F. Science \& Engineering Research Expenditures (\$M)

\begin{tabular}{|c|c|c|c|c|c|c|c|c|c|c|}
\hline & $2014-15$ & $2015-16$ & 2016-17 & $2017-18$ & 2018-19 & $2019-20$ & 2020-21 & $2021-22$ & $2022-23$ & 2023-24 \\
\hline ACTUAL & 420 & 448 & 502 & 525 & 331 & . & . & . & & . \\
\hline $\begin{array}{l}\text { RESTATED } \\
\text { ACTUALS }\end{array}$ & 284 & 305 & 325 & 335 & & . & & . & & . \\
\hline APPROVED GOALS & . & 421 & 427 & 503 & 530 & 545 & 560 & 575 & . & . \\
\hline PROPOSED GOALS & . & . & . & . & . & 333 & 334 & 336 & 338 & 339 \\
\hline
\end{tabular}

G. Non-Medical Science \& Engineering Research Expenditures (\$M)

\begin{tabular}{ccccccccccc} 
& $2014-15$ & $2015-16$ & $2016-17$ & $2017-18$ & $2018-19$ & $2019-20$ & $2020-21$ & $2021-22$ & $2022-23$ & $2023-24$ \\
\hline ACTUAL & 229 & 229 & 288 & 296 & 248 &. &. &. &. &. \\
$\begin{array}{c}\text { RESTATED } \\
\text { ACTUALS }\end{array}$ & 210 & 207 & 264 & 250 & &. &. &. &. &. \\
APPROVED GOALS &. & 230 & 233 & 289 & 296 & 300 & 305 & 310 &. &. \\
PROPOSED GOALS &. &. &. &. &. & 249 & 250 & 252 & 253 & 254
\end{tabular}

H. Number of Broad Disciplines Ranked in Top 100 for Research Expenditures

\begin{tabular}{cccccccccccc} 
& $2013-14$ & $2014-15$ & $2015-16$ & $2016-17$ & $2017-18$ & $2018-19$ & $2019-20$ & $2020-21$ & $2021-22$ & $2022-23$ \\
\hline ACTUAL & 7 & 7 & 7 & 7 & 7 &. &. &. &. &. \\
RESTATED & 7 & 7 & 7 & 7 & 7 &. &. &. & &. \\
ACTUALS & 7 & 7 of 8 & 8 of 8 & 8 of 8 & 8 of 8 & 8 of 8 & 8 of 8 & 8 of 8 &. &. \\
APPROVED GOALS &. &. &. &. &. & 8 of 8 & 8 of 8 & 8 of 8 & 8 of 8 & 8 of 8
\end{tabular}




\section{PREEMINENT RESEARCH UNIVERSITY FUNDING METRICS (cont.)}

I. Utility Patents Awarded [over three calendar years]

\begin{tabular}{cccccccccccc} 
& $2013-15$ & $2014-16$ & $2015-17$ & $2016-18$ & $2017-19$ & $2018-20$ & $2019-21$ & $2020-22$ & $2021-23$ & $2022-24$ \\
\hline ACTUAL & 298 & 314 & 324 & 335 & 338 &. &. &. &. &. \\
APPROVED GOALS &. & 291 & 273 & 325 & 323 & 306 & 309 & 312 &. &. \\
PROPOSED GOALS &. &. &. &. &. & 338 & 338 & 338 & 338 & 338
\end{tabular}

\section{J. Doctoral Degrees Awarded Annually}

\begin{tabular}{|c|c|c|c|c|c|c|c|c|c|c|}
\hline & 2014-15 & $2015-16$ & 2016-17 & $2017-18$ & 2018-19 & $2019-20$ & $2020-21$ & 2021-22 & $2022-23$ & 2023-24 \\
\hline ACTUAL & 601 & 704 & 721 & 701 & 752 & . & . & . & . & \\
\hline APPROVED GOALS & . & 645 & 650 & 725 & 717 & 720 & 725 & 725 & . & . \\
\hline PROPOSED GOALS & r & . & r & . & & 720 & 725 & 730 & 730 & 730 \\
\hline
\end{tabular}

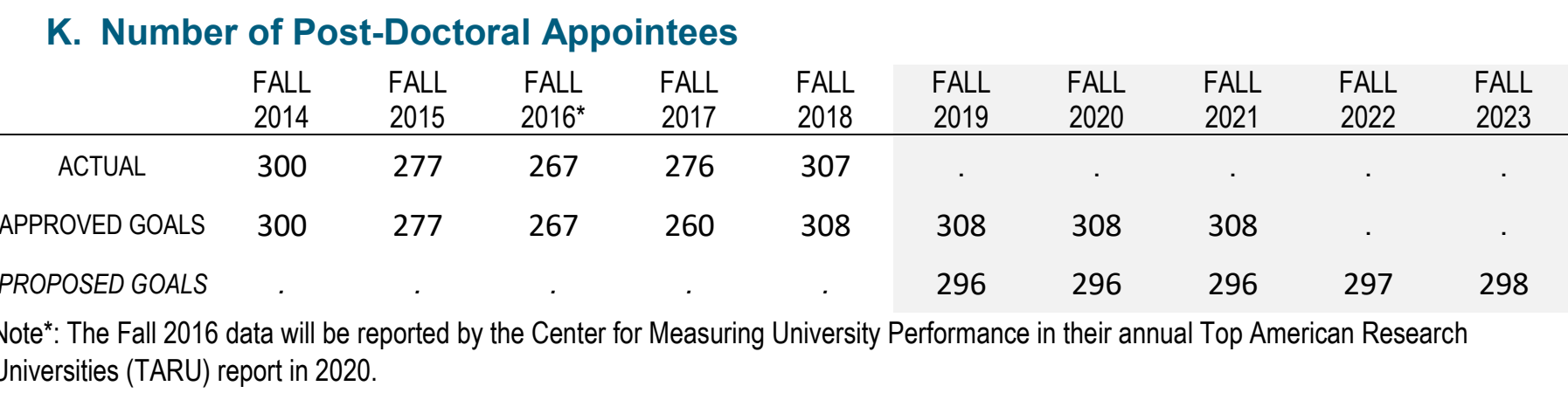

\begin{tabular}{lcccccccccc}
\multicolumn{2}{l}{ L. Endowment Size $(\$ \mathbf{M})$} & & & & & & & & \\
& $2014-15$ & $2015-16$ & $2016-17$ & $2017-18$ & $2018-19$ & $2019-20$ & $2020-21$ & $2021-22$ & $2022-23$ & $2023-24$ \\
\hline ACTUAL & 417 & 395 & 442 & 480 & 514 &. &. &. &. &. \\
APROVED GOALS &. & 395 & 412 & 450 & 466 & 486 & 505 & 525 &. &. \\
POPOSED GOALS &. &. &. &. &. & 465 & 500 & 520 & 550 & 575
\end{tabular}




\section{KEY PERFORMANCE INDICATORS}

Teaching \& Learning (from the 2025 System Strategic Plan not included in PBF section)

1. Public University National Ranking [Number of Top50 Rankings based on BOG's official list of publications]

\begin{tabular}{ccccccccccc} 
& 2016 & 2017 & 2018 & 2019 & 2020 & 2021 & 2022 & 2023 & 2024 & 2025 \\
\hline ACTUAL & 4 & 4 & 4 & 4 & 6 &. &. &. &. &. \\
APPROVED GOALS &. & 5 & 5 & 5 & 5 & 5 & 5 & 5 &. &. \\
PROPOSED GOALS &. &. &. &. &. & 6 & 6 & 6 & 7 & 7
\end{tabular}

2. Freshmen in Top $10 \%$ of High School Class

\begin{tabular}{|c|c|c|c|c|c|c|c|c|c|c|}
\hline & $\begin{array}{l}\text { FALL } \\
2015 \\
\end{array}$ & $\begin{array}{l}\text { FALL } \\
2016 \\
\end{array}$ & $\begin{array}{l}\text { FALL } \\
2017 \\
\end{array}$ & $\begin{array}{l}\text { FALL } \\
2018 \\
\end{array}$ & $\begin{array}{l}\text { FALL } \\
2019 \\
\end{array}$ & $\begin{array}{l}\text { FALL } \\
2020 \\
\end{array}$ & $\begin{array}{l}\text { FALL } \\
2021 \\
\end{array}$ & $\begin{array}{l}\text { FALL } \\
2022 \\
\end{array}$ & $\begin{array}{l}\text { FALL } \\
2023 \\
\end{array}$ & $\begin{array}{l}\text { FALL } \\
2024 \\
\end{array}$ \\
\hline ACTUAL & 30 & 33 & 34 & 32 & 33 & . & . & . & . & . \\
\hline APPROVED GOALS & . & 30 & 33 & 34 & 34.5 & 35 & 36 & 37 & . & . \\
\hline PROPOSED GOALS & . & . & . & . & . & 35 & 36 & 37 & 38 & 39 \\
\hline
\end{tabular}

\section{Time to Degree for FTICs in $120 \mathrm{hr}$ programs}

\begin{tabular}{ccccccccccc} 
& $2014-15$ & $2015-16$ & $2016-17$ & $2017-18$ & $2018-19$ & $2019-20$ & $2020-21$ & $2021-22$ & $2022-23$ & $2023-24$ \\
\hline ACTUAL & 4.5 & 4.3 & 4.2 & 4.0 & 4.0 &. &. &. &. &. \\
APPROVED GOALS &. & 4.5 & 4.3 & 4.2 & 4.0 & 4.0 & 4.0 & 4.0 &. &. \\
PROPOSED GOALS &. &. &. &. &. & 4.0 & 4.0 & 4.0 & 4.0 & 4.0
\end{tabular}

4. Six-Year FTIC Graduation Rates [Full-\& Part-time students]

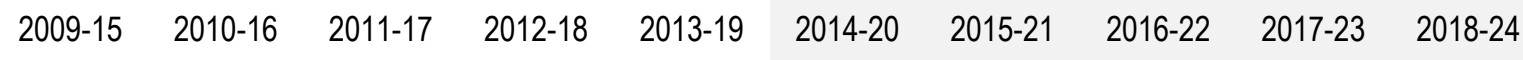

\begin{tabular}{cccc|c|c|c|c|c|c|c}
\hline ACTUAL & 68 & 66 & 69 & 71 & 73 &. &. &. &. &. \\
APPROVED GOALS &. & 66.7 & 70 & 69 & 71.3 & 72.1 & 72.1 & 73.3 &. &. \\
PROPOSED GOALS &. &. &. &. &. & 74.0 & 74.5 & 75.0 & 75.5 & 76.0
\end{tabular}

5. FCS AA Transfer Three-Year Graduation Rate (Florida College System w/ Associate in Arts)

\begin{tabular}{ccccccccccc} 
& $2012-15$ & $2013-16$ & $2014-17$ & $2015-18$ & $2016-19$ & $2017-20$ & $2018-21$ & $2019-22$ & $2020-23$ & $2021-24$ \\
\hline ACTUAL & 57 & 56 & 59 & 59 & 61 &. &. &. &. &. \\
APPROVED GOALS &. &. &. &. &. &. &. &. &. &. \\
PROPOSED GOALS &. &. &. &. &. & 61.0 & 61.2 & 61.7 & 62.2 & 62.7
\end{tabular}




\section{KEY PERFORMANCE INDICATORS (cont.)}

Teaching \& Learning (from the 2025 System Strategic Plan not included in PBF section)

6. Pell Recipient Four-Year Graduation Rate [for Full-Time FTIC]

\begin{tabular}{ccccccccccc} 
& $2011-15$ & $2012-16$ & $2013-17$ & $2014-18$ & $2015-19$ & $2016-20$ & $2017-21$ & $2018-22$ & $2019-23$ & $2020-24$ \\
\hline ACTUAL & 48 & 50 & 54 & 55 & 57 &. &. &. &. &. \\
APPROVED GOALS &. &. &. &. &. &. &. &. &. &. \\
PROPOSED GOALS &. &. &. &. &. & 57.3 & 59.6 & 60.0 & 60.5 & 61.0
\end{tabular}

\section{Bachelor's Degrees Awarded [First Majors Only]}

\begin{tabular}{ccccccccccc} 
& $2014-15$ & $2015-16$ & $2016-17$ & $2017-18$ & $2018-19$ & $2019-20$ & $2020-21$ & $2021-22$ & $2022-23$ & $2023-24$ \\
\hline ACTUAL & 9,290 & 9,222 & 9,503 & 9,679 & 10,007 &. &. &. &. &. \\
APPROVED GOALS &. & 9,081 & 9,255 & 9,513 & 9,765 & 9,842 & 9,923 & 10,044 &. &. \\
PROPOSED GOALS &. &. &. &. &. & 9,845 & 10,011 & 10,128 & 10,246 & 10,312
\end{tabular}

8. Graduate Degrees Awarded [First Majors Only]

\begin{tabular}{cccccc|ccccc} 
& $2014-15$ & $2015-16$ & $2016-17$ & $2017-18$ & $2018-19$ & $2019-20$ & $2020-21$ & $2021-22$ & $2022-23$ & $2023-24$ \\
\hline ACTUAL & 3,773 & 3,918 & 4,208 & 4,169 & 4,299 &. &. &. &. &. \\
APPROVED GOALS &. & 3,877 & 3,973 & 4,233 & 4,161 & 4,177 & 4,195 & 4,215 &. &. \\
PROPOSED GOALS &. &. &. &. &. & 4,239 & 4,267 & 4,286 & 4,315 & 4,334
\end{tabular}

9. Percentage of Bachelor's Degrees Awarded to African-American \& Hispanic Students

\begin{tabular}{cccccc|c|c|c|c|c} 
& $2014-15$ & $2015-16$ & $2016-17$ & $2017-18$ & $2018-19$ & $2019-20$ & $2020-21$ & $2021-22$ & $2022-23$ & $2023-24$ \\
\hline ACTUAL & 30 & 31 & 32 & 30 & 33 &. &. &. &. &. \\
APPROVED GOALS &. & 30 & 31 & 32 & 32.4 & 32.5 & 33.2 & 33.8 &. &. \\
PROPOSED GOALS &. &. &. &. &. & 33.0 & 33.5 & 34.0 & 34.5 & 35.0
\end{tabular}

10. Percentage of Adult (Aged 25+) Undergraduates Enrolled

\begin{tabular}{ccccccccccc} 
& FALL & FALL & FALL & FALL & FALL & FALL & FALL & FALL & FALL & FALL \\
& 2015 & 2016 & 2017 & 2018 & 2019 & 2020 & 2021 & 2022 & 2023 & 2024 \\
\hline ACTUAL & 23 & 22 & 22 & 20 & 19 &. &. &. &. &. \\
APPROVED GOALS &. & 23 & 22 & 20 & 20.5 & 20.8 & 20.8 & 20.9 &. &. \\
PROPOSED GOALS &. &. &. &. &. & 19 & 19 & 19 & 19 & 19
\end{tabular}




\section{KEY PERFORMANCE INDICATORS (cont.)}

Teaching \& Learning (from the 2025 System Strategic Plan not included in PBF section)

11. Percent of Undergraduate FTE in Online Courses

\begin{tabular}{|c|c|c|c|c|c|c|c|c|c|c|}
\hline & $2014-15$ & $2015-16$ & 2016-17 & $2017-18$ & $2018-19$ & $2019-20$ & $2020-21$ & $2021-22$ & $2022-23$ & 2023-24 \\
\hline ACTUAL & 26 & 28 & 29 & 32 & 32 & & & & & \\
\hline APPROVED GOALS & . & 28 & 28 & 30 & 32.6 & 33.7 & 33.7 & 35.4 & . & . \\
\hline PROPOSED GOALS & & . & . & . & . & 34 & 35 & 36 & 37 & 38 \\
\hline
\end{tabular}

12. Percent of Bachelor's Degrees in STEM \& Health

\begin{tabular}{ccccccccccc} 
& $2014-15$ & $2015-16$ & $2016-17$ & $2017-18$ & $2018-19$ & $2019-20$ & $2020-21$ & $2021-22$ & $2022-23$ & $2023-24$ \\
\hline ACTUAL & 38 & 42 & 44 & 45 & 46 &. &. &. &. &. \\
APPROVED GOALS &. & 41 & 42 & 44 & 46 & 48.7 & 49.8 & 50 &. &. \\
PROPOSED GOALS &. &. &. &. &. & 48.7 & 49.8 & 50 & 50 & 50
\end{tabular}

\section{Percent of Graduate Degrees in STEM \& Health}

\begin{tabular}{cccccccccccc} 
& $2014-15$ & $2015-16$ & $2016-17$ & $2017-18$ & $2018-19$ & $2019-20$ & $2020-21$ & $2021-22$ & $2022-23$ & $2023-24$ \\
\hline ACTUAL & 57 & 60 & 62 & 60 & 61 &. &. &. &. &. \\
APPROVED GOALS &. & 60 & 61 & 63 & 61 & 62 & 63 & 63 &. &. \\
PROPOSED GOALS &. &. &. &. &. & 62 & 63 & 63 & 63 & 63
\end{tabular}




\section{KEY PERFORMANCE INDICATORS (cont.)}

Teaching \& Learning (from the 2025 System Strategic Plan not included in PBF section)

14. Professional Licensure \& Certification Exam First-time Pass Rates

\begin{tabular}{lcccccccccc} 
CALENDAR YEAR & 2015 & 2016 & 2017 & 2018 & 2019 & 2020 & 2021 & 2022 & 2023 & 2024 \\
\hline NURSING & 90 & 94 & 93 & 94 & 94 & 94 & 94 & 94 & 94 & 94 \\
US Average & 87 & 88 & 90 & 92 & 91 &. &. &. &. &. \\
\hline MEDICINE (2YR) & 96 & 94 & 92 & 98 & 99 & 98 & 98 & 98 & 98 & 98 \\
US Average & 96 & 96 & 96 & 96 & 97 &. &. &. &. & $\cdot$ \\
\hline PHARMACY & 94 & 91 & 86 & 83 & 90 & 90 & 90 & 90 & 91 & 91 \\
US Average & 93 & 86 & 88 & 89 & 88 &. &. &. &. &. \\
CROSS-YEAR & $2014-15$ & $2015-16$ & $2016-17$ & $2017-18$ & $2018-19$ & $2019-20$ & $2020-21$ & $2021-22$ & $2022-23$ & $2023-24$ \\
\hline MEDICINE (4Y-CK) & 97 & 99 & 95 & 98 & 96 & 98 & 98 & 98 & 98 & 98 \\
US Average & 95 & 96 & 96 & 97 & 98 &. &. &. &. &. \\
MEDICINE (4Y-CS) & 96 & 97 & 96 & 97 & 94 & 97 & 97 & 98 & 98 & 98 \\
US Average & 96 & 97 & 96 & 95 & 95 &. &. &. &. &. \\
MULTI-YEAR & $2013-15$ & $2014-16$ & $2015-17$ & $2016-18$ & $2017-19$ & $2018-20$ & $2019-21$ & $2020-22$ & $2021-23$ & $2022-24$ \\
\hline PHYSICAL THERAPY & 95 & 94 & 95 & 94 & 93 & 93 & 93 & 93 & 93 & 93 \\
US Average & 91 & 92 & 92 & 92 & 92 &. &. &. &. &.
\end{tabular}

Exam Scores Relative to Benchmarks

\begin{tabular}{lcccccccccc} 
& 2015 & 2016 & 2017 & 2018 & 2019 & 2020 & 2021 & 2022 & 2023 & 2024 \\
\hline ABOVE OR TIED & 6 & 6 & 3 & 5 & 4 & 6 & 6 & 6 & 6 & 6 \\
TOTAL & 6 & 6 & 6 & 6 & 6 & 6 & 6 & 6 & 6 & 6
\end{tabular}




\section{KEY PERFORMANCE INDICATORS (cont.) \\ Scholarship, Research \& Innovation Metrics}

\section{National Academy Memberships}

\begin{tabular}{ccccccccccc} 
& 2016 & 2017 & 2018 & 2019 & 2020 & 2021 & 2022 & 2023 & 2024 & 2025 \\
\hline ACTUAL & 8 & 11 & 13 & 14 & 15 &. &. &. &. &. \\
APPROVED GOALS &. & 9 & 10 & 13 & 14 & 15 & 16 & 17 &. &. \\
PROPOSED GOALS &. &. &. &. &. & 15 & 16 & 17 & 17 & 17
\end{tabular}

16. Faculty Awards

\begin{tabular}{ccccccccccc} 
& FALL & FALL & FALL & FALL & FALL & FALL & FALL & FALL & FALL & FALL \\
& 2013 & 2014 & 2015 & 2016 & 2017 & 2018 & 2019 & 2020 & 2021 & 2022 \\
\hline ACTUAL & 8 & 8 & 13 & 13 & 10 &. &. &. &. &. \\
APPROVED GOALS &. & 7 & 8 & 9 & 12 & 12 & 12 & 13 &. &. \\
PROPOSED GOALS &. &. &. &. &. & 12 & 12 & 13 & 13 & 13
\end{tabular}

\section{Total Research Expenditures (\$M)}

\begin{tabular}{|c|c|c|c|c|c|c|c|c|c|c|}
\hline & 2014-15 & 2015-16 & 2016-17 & $2017-18$ & 2018-19 & $2019-20$ & $2020-21$ & $2021-22$ & $2022-23$ & 2023-24 \\
\hline ACTUAL & 494 & 515 & 568 & 592 & 405 & . & & . & & . \\
\hline RESTATED ACTUAL & $350 *$ & $365^{*}$ & $384 *$ & $396 * *$ & 405 & . & . & . & . & . \\
\hline APPROVED GOALS & . & 495 & 510 & 569 & 600 & 601 & 602 & 603 & . & . \\
\hline PROPOSED GOALS & . & . & . & . & . & 407 & 409 & 411 & 413 & 415 \\
\hline
\end{tabular}

\section{Research Expenditures from External Sources (\$M)}

\begin{tabular}{|c|c|c|c|c|c|c|c|c|c|c|}
\hline & 2014-15 & $2015-16$ & 2016-17 & $2017-18$ & 2018-19 & $2019-20$ & $2020-21$ & $2021-22$ & $2022-23$ & 2023-24 \\
\hline ACTUAL & 272 & 282 & 323 & 335 & 220 & . & $\cdot$ & . & & . \\
\hline RESTATED ACTUAL & $193 *$ & $200 *$ & $218^{*}$ & $221 * *$ & 220 & . & . & . & & . \\
\hline APPROVED GOALS & . & . & . & . & . & . & . & . & . & . \\
\hline PROPOSED GOALS & . & . & . & . & . & 221 & 222 & 223 & 224 & 226 \\
\hline $\begin{array}{l}\text { Note*: Previously } \\
\text { of research expen } \\
\text { other institutions } \\
\text { training grants. } \\
\text { variance between }\end{array}$ & $\begin{array}{l}\text { reported } \\
\text { ditures ass } \\
\text { (e.g., H. Le } \\
\text { Although } \\
\text { the data r }\end{array}$ & $\begin{array}{l}\text { ata for thi } \\
\text { ciated wi } \\
\text { Moffitt C } \\
\text { he Nation } \\
\text { ported in }\end{array}$ & $\begin{array}{l}\text { metric ha } \\
\text { h dually-a| } \\
\text { ncer Cent } \\
\text { I Science F } \\
\text { Y } 2018 \text { an }\end{array}$ & $\begin{array}{l}\text { oeen rest } \\
\text { ointed U } \\
\text { \& Resea } \\
\text { undation } \\
\text { FY 2019, }\end{array}$ & $\begin{array}{l}\text { ed to add } \\
\text { staff for } \\
\text { h Institute } \\
\text { JSF) does } \\
\text { e NSF req }\end{array}$ & $\begin{array}{l}\text { ess a metl } \\
\text { vork that } \\
\text { ), an alloc } \\
\text { lot require } \\
\text { uested tha }\end{array}$ & $\begin{array}{l}\text { odological } \\
\text { as include } \\
\text { tion of gra } \\
\text { modificati } \\
\text { USF resta }\end{array}$ & $\begin{array}{l}\text { error. This } \\
\text { on the fir } \\
\text { luate med } \\
\text { n of prior }\end{array}$ & $\begin{array}{l}\text { was largel } \\
\text { ancial stat } \\
\text { cal researc } \\
\text { years' data } \\
\text { ers for FY }\end{array}$ & $\begin{array}{l}\text { comprisec } \\
\text { ments at } \\
\text { effort, an } \\
\text { given the } \\
\text { J18 to }\end{array}$ \\
\hline
\end{tabular}




\section{KEY PERFORMANCE INDICATORS (cont.)}

Scholarship, Research \& Innovation Metrics

19. Utility Patents Awarded

\begin{tabular}{cccccccccccc} 
& 2015 & 2016 & 2017 & 2018 & 2019 & 2020 & 2021 & 2022 & 2023 & 2024 \\
\hline ACTUAL & 90 & 114 & 120 & 101 & 117 &. &. &. &. &. \\
APPROVED GOALS &. &. & 69 & 121 & 102 & 103 & 104 & 105 &. &. \\
PROPOSED GOALS &. &. &. &. &. & 119 & 120 & 121 & 122 & 123
\end{tabular}

20. Number of Licenses/Options Executed Annually

\begin{tabular}{cccccccccccc} 
& $2013-14$ & $2014-15$ & $2015-16$ & $2016-17$ & $2017-18$ & $2018-19$ & $2019-20$ & $2020-21$ & $2021-22$ & $2022-23$ \\
\hline ACTUAL & 91 & 119 & 133 & 122 & 127 &. &. &. &. & \\
APPROVED GOALS &. & 119 & 120 & 121 & 123 & 90 & 91 & 92 & 93 & \\
PROPOSED GOALS &. &. &. &. &. & 91 & 92 & 93 & 94 & 95
\end{tabular}

\section{Number of Start-up Companies Created}

\begin{tabular}{cccccccccccc} 
& $2013-14$ & $2014-15$ & $2015-16$ & $2016-17$ & $2017-18$ & $2018-19$ & $2019-20$ & $2020-21$ & $2021-22$ & $2022-23$ \\
\hline ACTUAL & 11 & 11 & 9 & 10 & 10 &. &. &. &. &. \\
APPROVED GOALS &. & 8 & 8 & 9 & 10 & 10 & 11 & 11 & 12 &. \\
PROPOSED GOALS &. &. &. &. &. & 6 & 8 & 9 & 10 & 11
\end{tabular}




\section{KEY PERFORMANCE INDICATORS (cont.) Institution Specific Goals}

To further distinguish the university's distinctive mission, the university may choose to provide additional metric goals that are based on the university's own strategic plan.

\section{Maintain the Normalized Citation Impact above 1.5}

\begin{tabular}{|c|c|c|c|c|c|c|c|c|c|c|}
\hline & $\begin{array}{l}2008 / 12- \\
2010 / 14 \\
\end{array}$ & $\begin{array}{l}2009 / 13- \\
2011 / 15 \\
\end{array}$ & $\begin{array}{l}2010 / 14- \\
2012 / 16 \\
\end{array}$ & $\begin{array}{l}2011 / 15- \\
2013 / 17 \\
\end{array}$ & $\begin{array}{l}2012 / 16- \\
2014 / 18 \\
\end{array}$ & $\begin{array}{l}2013 / 17- \\
2015 / 19 \\
\end{array}$ & $\begin{array}{l}2014 / 18- \\
2016 / 20 \\
\end{array}$ & $\begin{array}{l}2015 / 19- \\
2017 / 21 \\
\end{array}$ & $\begin{array}{l}2016 / 20- \\
2018 / 22 \\
\end{array}$ & $\begin{array}{l}2017 / 21- \\
2019 / 23 \\
\end{array}$ \\
\hline ACTUAL & 1.56 & 1.58 & 1.6 & 1.61 & 1.59 & & & & & r \\
\hline $\begin{array}{c}\text { APPROVED } \\
\text { GOALS }\end{array}$ & . & $\cdot$ & $\cdot$ & $\cdot$ & · & & · & & . & . \\
\hline $\begin{array}{c}\text { PROPOSED } \\
\text { GOALS }\end{array}$ & . & . & . & . & . & $\begin{array}{c}\text { Maintain } \\
\text { above } \\
1.5\end{array}$ & $\begin{array}{c}\text { Maintain } \\
\text { above } \\
1.5\end{array}$ & $\begin{array}{c}\text { Maintain } \\
\text { above } \\
1.5\end{array}$ & $\begin{array}{c}\text { Maintain } \\
\text { above } \\
1.5\end{array}$ & $\begin{array}{c}\text { Maintain } \\
\text { above } \\
1.5\end{array}$ \\
\hline
\end{tabular}

2. Percentage of undergraduate classes with fewer than 20 students.

\begin{tabular}{ccccccccccc} 
& Fall 2015 & Fall 2016 & Fall 2017 & Fall 2018 & Fall 2019 & Fall 2020 & Fall 2021 & Fall 2022 & Fall 2023 & Fall 2024 \\
\hline ACTUAL & 31 & 31 & 30 & 44 & 43 &. &. &. &. &. \\
APPROVED GOALS &. &. &. &. &. &. &. &. &. &. \\
PROPOSED GOALS &. &. &. &. &. & 44 & 46 & 48 & 50 & 52
\end{tabular}




\section{ENROLLMENT PLANNING}

Fall Headcount Enrollment by Student Level [all degree-seeking students, all campuses]

\begin{tabular}{|c|c|c|c|c|c|c|c|c|c|c|}
\hline UNDERGRADUATE & 2015 & 2016 & 2017 & 2018 & 2019 & 2020 & 2021 & 2022 & 2023 & 2024 \\
\hline ACTUAL & 35,990 & 36,373 & 36,955 & 37,235 & 37,284 & & & & . & . \\
\hline APPROVED GOALS & . & & 36,992 & 36,850 & 37,323 & 38,283 & 39,215 & 40,358 & $\cdot$ & $\cdot$ \\
\hline PROPOSED GOALS & . & & . & . & & 37,289 & 37,289 & 37,286 & 37,285 & 37,284 \\
\hline GRADUATE & 2015 & 2016 & 2017 & 2018 & 2019 & 2020 & 2021 & 2022 & 2023 & 2024 \\
\hline ACTUAL & 10,698 & 10,983 & 11,569 & 11,438 & 11,389 & & . & & . & . \\
\hline APPROVED GOALS & . & 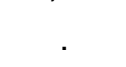 & 11,094 & 11,657 & 11,497 & 11,571 & 11,646 & 11,749 & . & . \\
\hline PROPOSED GOALS & . & & . & . & . & 11,411 & 11,495 & 11,584 & 11,638 & 11,669 \\
\hline
\end{tabular}

Fall Headcount Enrollment by Student Type [all degree-seeking students, all campuses]

\begin{tabular}{lcccccccccc} 
UNDERGRADUATE & 2015 & 2016 & 2017 & 2018 & 2019 & 2020 & 2021 & 2022 & 2023 & 2024 \\
\hline FTIC: New & 4,852 & 4,802 & 4,943 & 5,111 & 5,539 & 5,700 & 5,710 & 5,720 & 5,730 & 5,730 \\
FTIC: Returning & 12,852 & 13,221 & 13,246 & 13,464 & 13,634 & 13,690 & 13,750 & 13,782 & 13,760 & 13,764 \\
Transfer: FCS w/ AA & 9,108 & 9,245 & 9,416 & 9,501 & 9,271 & 9,316 & 9,350 & 9,356 & 9,389 & 9,407 \\
Transfer: Other & 8,153 & 8,107 & 8,364 & 8,258 & 7,864 & 7,596 & 7,492 & 7,441 & 7,419 & 7,396 \\
Post-Baccalaureates & 1,025 & 998 & 986 & 901 & 976 & 987 & 987 & 987 & 987 & 987 \\
Subtotal & $\mathbf{3 5 , 9 9 0}$ & $\mathbf{3 6 , 3 7 3}$ & $\mathbf{3 6 , 9 5 5}$ & $\mathbf{3 7 , 2 3 5}$ & $\mathbf{3 7 , 2 8 4}$ & $\mathbf{3 7 , 2 8 9}$ & $\mathbf{3 7 , 2 8 9}$ & $\mathbf{3 7 , 2 8 6}$ & $\mathbf{3 7 , 2 8 5}$ & $\mathbf{3 7 , 2 8 4}$ \\
& & & & & & & & & & \\
GRADUATE & 2015 & 2016 & 2017 & 2018 & 2019 & 2020 & 2021 & 2022 & 2023 & 2024 \\
\hline Master's & $\mathbf{7 , 1 6 0}$ & $\mathbf{7 , 3 0 2}$ & $\mathbf{7 , 6 9 0}$ & $\mathbf{7 , 4 8 9}$ & $\mathbf{7 , 4 6 3}$ & $\mathbf{7 , 4 8 9}$ & $\mathbf{7 , 5 5 5}$ & $\mathbf{7 , 6 2 5}$ & $\mathbf{7 , 6 6 8}$ & $\mathbf{7 , 6 9 2}$ \\
Research Doctoral & $\mathbf{2 , 2 2 9}$ & $\mathbf{2 , 3 3 3}$ & $\mathbf{2 , 4 4 3}$ & $\mathbf{2 , 4 6 1}$ & $\mathbf{2 , 4 1 7}$ & $\mathbf{2 , 4 2 2}$ & $\mathbf{2 , 4 4 0}$ & $\mathbf{2 , 4 5 9}$ & $\mathbf{2 , 4 7 0}$ & $\mathbf{2 , 4 7 7}$ \\
Professional Doctoral & $\mathbf{1 , 3 0 9}$ & $\mathbf{1 , 3 4 8}$ & $\mathbf{1 , 4 3 6}$ & $\mathbf{1 , 4 8 8}$ & $\mathbf{1 , 5 0 9}$ & $\mathbf{1 , 5 0 0}$ & $\mathbf{1 , 5 0 0}$ & $\mathbf{1 , 5 0 0}$ & $\mathbf{1 , 5 0 0}$ & $\mathbf{1 , 5 0 0}$ \\
Subtotal & $\mathbf{1 0 , 6 9 8}$ & $\mathbf{1 0 , 9 8 3}$ & $\mathbf{1 1 , 5 6 9}$ & $\mathbf{1 1 , 4 3 8}$ & $\mathbf{1 1 , 3 8 9}$ & $\mathbf{1 1 , 4 1 1}$ & $\mathbf{1 1 , 4 9 5}$ & $\mathbf{1 1 , 5 8 4}$ & $\mathbf{1 1 , 6 3 8}$ & $\mathbf{1 1 , 6 6 9}$ \\
TOTAL & $\mathbf{4 6 , 6 8 8}$ & $\mathbf{4 7 , 3 5 6}$ & $\mathbf{4 8 , 5 2 4}$ & $\mathbf{4 8 , 6 7 3}$ & $\mathbf{4 8 , 6 7 3}$ & $\mathbf{4 8 , 7 0 0}$ & $\mathbf{4 8 , 7 8 4}$ & $\mathbf{4 8 , 8 7 0}$ & $\mathbf{4 8 , 9 2 3}$ & $\mathbf{4 8 , 9 5 3}$
\end{tabular}

Note: This table reports this number of students enrolled by student type categories. These headcounts only include those seeking a degree unclassified students (eg, dual enrolled) are not included. The student type for undergraduates is based on the 'Type of Student at Most Recent Admission'. The First Time in College (FTIC) student was admitted in the same fall term or in the preceding summer term - this includes those who were re-admitted as FTICs. 


\section{ENROLLMENT PLANNING (cont.)}

Percent of Baccalaureate-Seeking Resident Undergraduates Earning 15+ Credits [Fall term]

\begin{tabular}{ccccccccccc} 
& 2015 & 2016 & 2017 & 2018 & 2019 & 2020 & 2021 & 2022 & 2023 & 2024 \\
\hline ACTUAL & 23 & 21 & 21 & 20 & 20 &. &. &. &. &. \\
APPROVED GOALS &. &. &. &. & 20 & 21 & 22 & 24 &. &. \\
PROPOSED GOALS &. &. &. &. &. & 21 & 22 & 24 & 24 & 24
\end{tabular}

Full-Time Equivalent (FTE) Enrollment by Course Level

\begin{tabular}{lcccccccccc} 
& $2014-15$ & $2015-16$ & $2016-17$ & $2017-18$ & $2018-19$ & $2019-20$ & $2020-21$ & $2021-22$ & $2022-23$ & $2023-24$ \\
\hline LOWER & 13,419 & 13,795 & 14,057 & 14,041 & 13,971 & 13,831 & 13,726 & 13,778 & 13,831 & 13,831 \\
UPPER & 20,262 & 20,423 & 20,158 & 20,796 & 21,021 & 21,456 & 21,456 & 21,456 & 21,500 & 21,537 \\
GRAD 1 & 6,396 & 6,553 & 6,482 & 6,534 & 6,404 & 6,278 & 6,304 & 6,316 & 6,341 & 6,314 \\
GRAD 2 & 2,159 & 2,134 & 2,236 & 2,385 & 2,452 & 2,449 & 2,551 & 2,617 & 2,684 & 2,750 \\
TOTAL & $\mathbf{4 2 , 2 3 6}$ & $\mathbf{4 2 , 9 0 5}$ & $\mathbf{4 2 , 9 3 2}$ & $\mathbf{4 3 , 7 5 6}$ & $\mathbf{4 3 , 8 4 9}$ & $\mathbf{4 4 , 0 1 4}$ & $\mathbf{4 4 , 0 3 7}$ & $\mathbf{4 4 , 1 6 7}$ & $\mathbf{4 4 , 3 5 6}$ & $\mathbf{4 4 , 4 3 2}$
\end{tabular}

Note: Full-time Equivalent (FTE) student is a measure of all instructional activity (regardless of fundability) that is based on the number of credit hours for all students during an academic (summer, fall, spring) year. FTE is based on the standard national definition, which divides undergraduate credit hours by 30 and graduate credit hours by 24. Pursuant to section 1013.31, Florida Statutes, Board facilities staff use this data as a key factor in the calculation of facility space needs for university educational plant surveys.

Percent FTE Enrollment by Method of Instruction

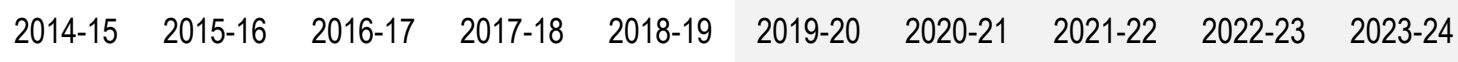

\section{UNDERGRADUATE}

All Distance (100\%)

Primarily Dist. (80-99\%)

Hybrid (50-79\%)

Classroom (0-49\%)

\section{GRADUATE}

All Distance $(100 \%)$

Primarily Dist. (80-99\%)

Hybrid (50-79\%)

Classroom (0-49\%)

$\begin{array}{cc}26 & 27 \\ 2 & 2 \\ 1 & 1 \\ 72 & 70\end{array}$

$27 \quad 30$

2

1

67

31
2
1
67

32
2
1

33

34

35

36

2

2

2

2

2

1

1

1

1

1

65

64

63

62

61 


\section{ACADEMIC PROGRAM COORDINATION}

\section{New Programs for Consideration by Institution in AY 2020-21}

The SUS Council of Academic Vice Presidents Academic Program Coordination Work Group will review these programs as part of their on-going coordination efforts. The programs listed below are based on the 2019 Accountability Plan list for programs under consideration for 2020-21.

\begin{tabular}{|c|c|c|c|c|c|c|}
\hline PROGRAM TITLES & CIP CODE & $\begin{array}{l}\text { AREA OF } \\
\text { STRATEGIC } \\
\text { EMPHASIS }\end{array}$ & $\begin{array}{l}\text { OTHER INST } \\
\text { W/ SAME } \\
\text { PROGRAM }\end{array}$ & $\begin{array}{c}\text { OFFERED } \\
\text { VIA } \\
\text { DISTANCE } \\
\text { LEARNING IN } \\
\text { SYSTEM } \\
\end{array}$ & $\begin{array}{c}\text { PROJECTED } \\
\text { ENROLLMENT IN } \\
5^{\text {TH }} \text { YEAR }\end{array}$ & $\begin{array}{l}\text { PROPOSED } \\
\text { DATE OF } \\
\text { SUBMISSION } \\
\text { TO UBOT }\end{array}$ \\
\hline \multicolumn{7}{|l|}{ UNDERGRADUATE } \\
\hline Environmental Engineering & 14.1401 & STEM & $\begin{array}{l}\text { FAU, FGCU, } \\
\text { FIU, FPU, } \\
\text { UCF, UF }\end{array}$ & $0 \%$ & 200 & Fall 2020 \\
\hline $\begin{array}{l}\text { Econometrics and } \\
\text { Quantitative Economics }\end{array}$ & 45.0603 & STEM & None & $0 \%$ & 200 & Spring 2021 \\
\hline
\end{tabular}

\section{MASTER'S, SPECIALIST AND OTHER ADVANCED MASTER'S PROGRAMS}

None

\section{DOCTORAL PROGRAMS}

None

New Programs for Consideration by Institution in AY 2021-22

These programs will be used in the 2021 Accountability Plan list for programs under consideration for 2021-22.

\begin{tabular}{|c|c|c|c|c|c|c|}
\hline & & & & OFFERED & & \\
\hline PROGRAM TITLES & $\begin{array}{c}\text { CIP } \\
\text { CODE }\end{array}$ & $\begin{array}{l}\text { AREA OF } \\
\text { STRATEGIC } \\
\text { EMPHASIS }\end{array}$ & $\begin{array}{l}\text { OTHER INST W/ } \\
\text { SAME } \\
\text { PROGRAM }\end{array}$ & $\begin{array}{c}\text { VIA } \\
\text { DISTANCE } \\
\text { LEARNING } \\
\text { IN } \\
\text { SYSTEM }\end{array}$ & $\begin{array}{l}\text { PROJECTED } \\
\text { ENROLLMENT } \\
\text { IN } 5^{\text {TH }} \text { YEAR }\end{array}$ & $\begin{array}{l}\text { PROPOSED } \\
\text { DATE OF } \\
\text { SUBMISSION } \\
\text { TO UBOT }\end{array}$ \\
\hline
\end{tabular}

\section{UNDERGRADUATE}

\begin{tabular}{lcccccc}
\hline Design & 50.0499 & None & None & $20 \%$ & 70 & Spring 2022 \\
Management Science & 52.1301 & STEM & FIU, FPU, UF & $100 \%$ & 50 & Fall 2021
\end{tabular}

\section{MASTER'S, SPECIALIST AND OTHER ADVANCED MASTER'S PROGRAMS}

\begin{tabular}{lllllll} 
Applied Mathematics & 27.0301 & STEM & $\begin{array}{l}\text { FAU, FGCU, FIU, } \\
\text { UCF, UNF }\end{array}$ & $0 \%$ & 35 & Fall 2021 \\
\hline $\begin{array}{l}\text { Personal Financial Planning } \\
\text { DOCTORAL PROGRAMS }\end{array}$ & 52.0804 & GAP & None & $20 \%$ & 40 & Spring 2022 \\
\begin{tabular}{lllll} 
Pharmacy (Ph.D.) \\
\hline
\end{tabular} & 51.2099 & Health & FAMU & $0 \%$ & 20 & TBD
\end{tabular}




\section{DEFINITIONS}

\section{Performance Based Funding (PBF)}

\section{PBF-1. Percent of Bachelor's Graduates Enrolled or Employed $(\mathbf{\$ 2 5 , 0 0 0 + )}$ One Year After Graduation:}

This metric is based on the percentage of a graduating class of bachelor's degree recipients who are enrolled or employed (earning at least $\$ 25,000$ ) somewhere in the United States. Students who do not have valid social security numbers and are not found enrolled are excluded. This data now includes: non-Florida data from 44 states and districts, including the District of Columbia and Puerto Rico; and military enlistment as reported by the institutions. Sources: State University Database System (SUDS), Florida Department of Economic Opportunity (DEO) analysis of Wage Record Interchange System (WRIS2), and National Student Clearinghouse (NSC).

\section{PBF-2. Median Wages of Bachelor's Graduates Employed Full-time One Year After Graduation}

This metric is based on annualized Unemployment Insurance (UI) wage data from the fourth fiscal quarter after graduation for bachelor's recipients. This data does not include individuals who are self-employed, employed by the military, those without a valid social security number, or making less than minimum wage. This data now includes non-Florida data from 44 states and districts, including the District of Columbia and Puerto Rico. State University Database System (SUDS), Florida Department of Economic Opportunity (DEO) analysis of Wage Record Interchange System (WRIS2).

\section{PBF-3. Cost to the Student Net Tuition \& Fees for Resident Undergraduates per $\mathbf{1 2 0}$ Credit Hours}

This metric compares the average sticker price and the average gift aid amount. The sticker price includes: (1) tuition and fees for resident undergraduates; (2) books and supplies (we use a proxy as calculated by the College Board); and (3) the average number of credit hours attempted by students who were admitted as an FTIC student who graduated with a bachelor's degree from a program that requires only 120 credit hours. The gift aid amount includes: (1) financial aid (grants, scholarships, waivers and third-party payments) provided to resident undergraduate students during the most recent academic year; (2) the total number of credit hours for those resident undergraduates. The average gift aid award per credit hour was multiplied by 120 and compared to the sticker price. Source: State University Database System (SUDS), the Legislature's annual General Appropriations Act, and university required fees.

\section{PBF-4. Four Year FTIC Graduation Rate}

This metric is based on the percentage of first-time-in-college (FTIC) students who started in the Fall (or summer continuing to Fall) term and were enrolled full-time in their first semester and had graduated from the same institution by the summer term of their fourth year. FTIC includes 'early admit' students who were admitted as a degree-seeking student prior to high school graduation. Students who were enrolled in advanced graduate programs during their $4^{\text {th }}$ year were excluded. Source: State University Database System (SUDS).

\section{PBF-5. Academic Progress Rate [2nd Year Retention with 2.0 GPA or Above]}

This metric is based on the percentage of first-time-in-college (FTIC) students who started in the Fall (or summer continuing to Fall) term and were enrolled full-time in their first semester and were still enrolled in the same institution during the next Fall term with a grade point average (GPA) of at least 2.0 at the end of their first year (Fall, Spring, Summer). Source: State University Database System (SUDS). 


\section{DEFINITIONS (cont.)}

\section{PBF-6. Bachelor's Degrees within Programs of Strategic Emphasis}

This metric is based on the number of baccalaureate degrees awarded within the programs designated by the Board of Governors as 'Programs of Strategic Emphasis'. A student who has multiple majors in the subset of targeted Classification of Instruction Program codes will be counted twice (i.e., double-majors are included). Source: State University Database System (SUDS).

\section{PBF-7. University Access Rate Percent of Undergraduates with a Pell-grant}

This metric is based the number of undergraduates, enrolled during the fall term, who received a Pell-grant during the fall term. Students who were not eligible for Pell-grants (e.g., Unclassified, non-resident aliens, post-baccs) were excluded from the denominator for this metric. Source: State University Database System (SUDS).

\section{PBF-8a. Graduate Degrees within Programs of Strategic Emphasis}

This metric is based on the number of graduate degrees awarded within the programs designated by the Board of Governors as 'Programs of Strategic Emphasis'. A student who has multiple majors in the subset of targeted Classification of Instruction Program codes will be counted twice (i.e., double-majors are included). Source: State University Database System (SUDS).

PBF-8b. Freshmen in Top $\mathbf{1 0 \%}$ of High School Class (Applies only to NCF)

Percent of all degree-seeking, first-time, first-year (freshman) students who had high school class rank within the top $10 \%$ of their graduating high school class. Source: New College of Florida as reported to the Common Data Set.

\section{PBF-9. Percent of Bachelor's Degrees Without Excess Hours}

This metric is based on the percentage of baccalaureate degrees awarded within $110 \%$ of the credit hours required for a degree based on the Board of Governors Academic Program Inventory. This metric excludes the following types of student credits (ie, accelerated mechanisms, remedial coursework, non-native credit hours that are not used toward the degree, non-native credit hours from failed, incomplete, withdrawn, or repeated courses, credit hours from internship programs, credit hours up to 10 foreign language credit hours, and credit hours earned in military science courses that are part of the Reserve Officers' Training Corps (ROTC) program). Starting in 2018-19, the calculation for this metric included a new type of statutory exclusion of up to 12 credit hours for students who graduated in four years or less. Source: State University Database System (SUDS).

Note: This metric does not report the number of students who paid the "Excess Hour Surcharge" (1009.286, FS).

PBF-10.FAMU: Number of Bachelor's Degrees Awarded to Transfers with AA Degrees from FCS: This is a count of first-major baccalaureate degrees awarded to students who entered as FCS AA Transfers. First Majors include the most common scenario of one student earning one degree in one Classification of Instructional Programs (CIP) code. A student who earns two baccalaureate degrees under two different degree CIPs is counted twice. Source: State University Database System (SUDS). 


\section{DEFINITIONS (cont.)}

PBF-10.FAU: Total Research Expenditures (\$M): Total expenditures (in millions of dollars) for all research activities (including non-science and engineering activities). Source: As reported by each institution to the National Science Foundation annual survey of Higher Education Research and Development (HERD) based on the NSF rules and definitions.

PBF-10.FGCU: Number of Bachelor's Degrees Awarded to Hispanic \& African-Americans: Race/Ethnicity data is selfreported by students. Non-Hispanic Black and Hispanic do not include students classified as Non-Resident Alien or students with a missing race code. Degree data is based on first-major counts only - second majors are not included.

PBF-10.FIU: Number of Post-Doctoral Appointees: The number of Postdoctoral Appointees awarded annually. This data is based on National Science Foundation/National Institutes of Health Survey of Graduate Students and Postdoctorates in Science and Engineering (GSS).

PBF-10.FPOLY: Percent of Bachelor's Graduates with 2+ Workforce Experiences: The percentage of Bachelor's recipients who completed at least two workforce experiences. Workforce experiences includes: External Internships, Industry-sponsored Capstone Projects, and Undergraduate Research (students on a funded research grant), and certifications. It is a requirement for all majors to conduct an external internship prior to graduation.

PBF-10.FSU: Percent of Bachelor's Graduates who took an Entrepreneurship Class: The percentage of Bachelor's recipients who enrolled in one or more graded Entrepreneurship courses before graduating.

PBF-10.NCF: Percent of FTIC Graduates Completing 3+ HIP's: The percentage of graduating seniors who started as FTIC students and who completing three or more high-impact practices as defined by the National Survey of Student Engagement (NSSE) and the Association of American Colleges \& Universities. High-impact practices include: (1) capstone project or thesis, (2) internships, (3) study abroad, (4) writing-intensive courses, (5) living-learning communities, (6) undergraduate research, (7) first-year experience, (8) learning communities, (9) service learning, (10) collaborative projects. Multiple activities within the same category only count once (e.g., a student completing three internships has completed one high impact practice).

PBF\#10.UCF: Percent of Bachelor's Degrees Awarded to African American and Hispanic Students: Percentage of Degrees is based on the number of baccalaureate degrees awarded to non-Hispanic Black and Hispanic students divided by the total degrees awarded - excluding those awarded to non-resident aliens and unreported. Source: State University Database System (SUDS).

PBF-10.UF: 6-Year Graduation Rates (FT only): The First-time-in-college (FTIC) cohort is defined as undergraduates entering in fall term (or summer continuing to fall) with fewer than 12 hours earned since high school graduation. The rate is the percentage of the initial cohort that has either graduated from the same institution by the summer term of their sixth academic year. Only full-time students are included in this calculation. FTIC includes 'early admits' students who were admitted as a degree-seeking student prior to high school graduation. Source: State University Database System (SUDS). 


\section{DEFINITIONS (cont.)}

PBF-10.UNF: Percent of Undergraduate FTE in Online Courses: Full-time Equivalent (FTE) student is a measure of instructional activity that is based on the number of credit hours that students enroll. FTE is based on the US definition, which divides undergraduate credit hours by 30. Distance Learning is a course in which at least 80 percent of the direct instruction of the course is delivered using some form of technology when the student and instructor are separated by time or space, or both (per 1009.24(17), F.S.). Source: State University Database System (SUDS).

PBF-10.USF: 6-Year Graduation Rates (FT/PT): The First-time-in-college (FTIC) cohort is defined as undergraduates entering in fall term (or summer continuing to fall) with fewer than 12 hours earned since high school graduation. The rate is the percentage of the initial cohort that has either graduated from the same institution by the summer term of their sixth academic year. Both full-time and part-time students are used in the calculation. FTIC includes 'early admits' students who were admitted as a degree-seeking student prior to high school graduation. Source: State University Database System (SUDS).

PBF-10.UWF: Percent of Baccalaureate Graduates Completing 2+ Types of High-Impact Practices: The percentage of graduating seniors completing two or more high-impact practices as defined by the Association of American Colleges \& Universities. High-impact practices include: (1) First Year Seminar \& Experiences, (2) Common Intellectual Experience, (3) Writing-Intensive Courses, (4) Collab Assignments \& Projects, (5) Diversity/Global Learning, (6) ePortolios, (7) Service Learning, Community-Based Learning, (8) Internships, (9) Capstone Courses \& Projects. Multiple activities within the same category only count once (e.g., a student completing three internships has completed one high impact practice).

\section{Preeminence Research University (PRE)}

PRE-A: Average GPA \& Average SAT: An average weighted grade point average of 4.0 or higher and an average SAT score of 1200 or higher for fall semester incoming freshmen, as reported annually in the admissions data that universities submit to the Board of Governors. This data includes registered FTIC (student type='B','E') with an admission action of admitted or provisionally admitted ('A',' $P$ ',' $X '$ '). Source: State University Database System (SUDS).

PRE-B: National University Rankings: A top-50 ranking on at least two well-known and highly respected national public university rankings, reflecting national preeminence, using most recent rankings, includes: Princeton Review, Fiske Guide, QS World University Ranking, Times Higher Education World University Ranking, Academic Ranking of World University, US News and World Report National University, US News and World Report National Public University, US News and World Report Liberal Arts Colleges, Forbes, Kiplinger, Washington Monthly Liberal Arts Colleges, Washington Monthly National University, and Center for Measuring University Performance.

PRE-C: Freshmen Retention Rate: Freshman Retention Rate (Full-time, FTIC) cohorts are based on first-year undergraduate students who enter the institution in the Fall term (or Summer term and continue into the Fall term). Percent retained is based on those who are enrolled during the second fall term. Source: State University Database System (SUDS) and data submitted by the institutions to Integrated Postsecondary Education Data System (IPEDS). 


\section{DEFINITIONS (cont.)}

PRE-D: 4-year Graduation Rate: This metric is based on the percentage of first-time-in-college (FTIC) students who started in the Fall (or summer continuing to Fall) term and were enrolled full-time in their first semester and had graduated from the same institution by the summer term of their fourth year. FTIC includes 'early admit' students who were admitted as a degree-seeking student prior to high school graduation. Students who were enrolled in advanced graduate programs during their $4^{\text {th }}$ year were excluded. Source: State University Database System (SUDS) and data submitted by the institutions to Integrated Postsecondary Education Data System (IPEDS).

PRE-E: National Academy Memberships: National Academy Memberships held by faculty as reported by the Center for Measuring University Performance in the Top American Research Universities (TARU) annual report or the official membership directories maintained by each national academy.

PRE-F: Total Science \& Engineering Research Expenditures: Research Expenditures within Science \& Engineering disciplines. Source: As reported by each institution to the National Science Foundation annual survey of Higher Education Research and Development (HERD) based on the NSF rules and definitions.

PRE-G: Science \& Engineering Research Expenditures in Non-Health Sciences: Research expenditures within Science \& Engineering in non-medical sciences. Source: As reported by each institution to the National Science Foundation annual survey of Higher Education Research and Development (HERD) based on the NSF rules and definitions.

PRE-H: National Ranking in Research Expenditures: The NSF identifies 8 broad disciplines within Science \& Engineering (Computer Science, Engineering, Environmental Science, Life Science, Mathematical Sciences, Physical Sciences, Psychology, Social Sciences). The rankings by discipline are determined by BOG staff using the NSF online database.

PRE-I: Patents Awarded: Total utility patents awarded by the United States Patent and Trademark Office (USPTO) for the most recent three calendar year period. Based on legislative staff guidance, Board staff query the USPTO database with a query that only counts utility patents: "(AN/"University Name" AND ISD/yyyymmdd->yyyymmdd AND APT/1)".

PRE-J: Doctoral Degrees Awarded Annually: Includes Doctoral research degrees and professional doctoral degrees awarded in medical and health care disciplines. Source: State University Database System (SUDS).

PRE-K: Number of Post-Doctoral Appointees: The number of Postdoctoral Appointees awarded annually. This data is based on National Science Foundation/National Institutes of Health Survey of Graduate Students and Postdoctorates in Science and Engineering (GSS).

PRE-L: Endowment Size (\$M): This data comes from the National Association of College and University Business Officers (NACUBO) and Commonfund Institute's annual report of Market Value of Endowment Assets.

\section{Key Performance Indicators (KPI)}

KPI-1: Public University National Ranking: A top-50 ranking on at least two well-known and highly respected national public university rankings, reflecting national preeminence, using most recent rankings, includes: Princeton Review, Fiske Guide, QS World University Ranking, Times Higher Education World University Ranking, Academic Ranking of World University, US News and World Report National University, US News and World Report National Public University, US News and World Report Liberal Arts Colleges, Forbes, Kiplinger, Washington Monthly Liberal Arts Colleges, Washington Monthly National University, and Center for Measuring University Performance. 


\section{DEFINITIONS (cont.)}

KPI-2: Freshmen in Top 10\% of High School Class: Percent of all degree-seeking, first-time, first-year (freshman) students who had high school class rank within the top 10\% of their graduating high school class. Source: As reported by the university to the Common Data Set.

KPI-3: Time to Degree for FTICs in 120hr programs: This metric is the number of years between the start date (using the student entry date) and the end date (using the last month in the term degree was granted) for a graduating class of first-time, single-major baccalaureates in 120 credit hour programs within a (Summer, Fall, Spring) year. Source: State University Database System (SUDS).

KPI-4: Six-Year FTIC Graduation Rates [full-\& part-time students]: The First-time-in-college (FTIC) cohort is defined as undergraduates entering in fall term (or summer continuing to fall) with fewer than 12 hours earned since high school graduation. The rate is the percentage of the initial cohort that has either graduated from the same institution by the summer term of their sixth academic year. Both full-time and part-time students are used in the calculation. FTIC includes 'early admits' students who were admitted as a degree-seeking student prior to high school graduation. Source: State University Database System (SUDS).

KPI-5: FCS AA Transfer Three-Year Graduation Rate [full-\& part-time students]: This transfer cohort is defined as undergraduates entering in fall term (or summer continuing to fall) from the Florida College System with an Associate in Arts (AA) degree. The rate is the percentage of the initial cohort that has either graduated from the same institution by the summer term of their third academic year. Both full-time and part-time students are used in the calculation. Students who were flagged as enrolled in advanced graduate programs that would not earn a bachelor's degree were not excluded. Source: State University Database System (SUDS).

KPI-6: Pell Recipient Four-Year Graduation Rate [for Full-Time FTIC]: This metric is based on the percentage of firsttime-in-college (FTIC) students who started in the Fall (or summer continuing to Fall) term and were enrolled full-time in their first semester and who received a Pell grant during their first year and who graduated from the same institution by the summer term of their fourth year. FTIC includes 'early admit' students who were admitted as a degree-seeking student prior to high school graduation. Students who were flagged as enrolled in advanced graduate programs that would not earn a bachelor's degree were excluded. Source: State University Database System (SUDS).

KPI-7: Bachelor's Degrees Awarded \& KPI-8: Graduate Degrees Awarded: This is a count of first-major baccalaureate and graduate degrees awarded. First Majors include the most common scenario of one student earning one degree in one Classification of Instructional Programs (CIP) code. In cases where a student earns a baccalaureate degree under two different degree CIPs, a distinction is made between "dual degrees" and "dual majors." Also included in first majors are "dual degrees" which are counted as separate degrees (e.g., counted twice). In these cases, both degree CIPs receive a "degree fraction" of 1.0. The calculation of degree fractions is made according to each institution's criteria. Source: State University Database System (SUDS).

KPI-9: Bachelor's Degrees Awarded to African-American \& Hispanic Students: Race/Ethnicity data is self-reported by students. Non-Hispanic Black and Hispanic do not include students classified as Non-Resident Alien or students with a missing race code. Degree data is based on first-major counts only - second majors are not included. Percentage of Degrees is based on the number of baccalaureate degrees awarded to non-Hispanic Black and Hispanic students divided by the total degrees awarded - excluding those awarded to non-resident aliens and unreported. Source: State University Database System (SUDS). 


\section{DEFINITIONS (cont.)}

KPI-10: Percentage of Adult (Aged 25+) Undergraduates Enrolled: This metric is based on the age of the student at the time of their Fall term enrollment - not their age upon entry. As a proxy, age is based on birth year not birth date. Note: Unclassified students with a HS diploma (or GED) and above are included in this calculation. Source: State University Database System (SUDS).

KPI-11: Percent of Undergraduate FTE in Online Courses: Full-time Equivalent (FTE) student is a measure of instructional activity that is based on the number of credit hours that students enroll. FTE is based on the US definition, which divides undergraduate credit hours by 30. Distance Learning is a course in which at least 80 percent of the direct instruction of the course is delivered using some form of technology when the student and instructor are separated by time or space, or both (per 1009.24(17), F.S.). Source: State University Database System (SUDS).

\section{KPI-12: Percent of Bachelor's Degrees in STEM \& Health \& KPI-13: Percent of Graduate Degrees in STEM \& Health:}

The percentage of baccalaureate degrees that are classified as STEM or Health disciplines by the Board of Governors in the Academic Program Inventory. These counts include second majors. Second Majors include all dual/second majors (e.g., degree CIP receive a degree fraction that is less than 1). The calculation of degree fractions is made according to each institution's criteria. The calculation for the number of second majors rounds each degree CIP's fraction of a degree up to 1 and then sums the total. Second Majors are typically used when providing degree information by discipline/CIP, to better conveys the number of graduates who have specific skill sets associated with each discipline. Source: State University Database System (SUDS).

KPI-14: Licensure \& Certification Exam Pass Rates: The average pass rates as a percentage of all first-time examinees for Nursing, Law, Medicine (3 subtests), Veterinary, Pharmacy, Dental (2 subtests), Physical Therapy, and Occupational Therapy, when applicable. The average pass rate for the nation or state is also provided as a contextual benchmark. The Board's 2025 System Strategic Plan calls for all institutions to be above or tied the exam's respective benchmark. The State benchmark for the Florida Bar Exam excludes non-Florida institutions. The national benchmark for the USMLE exams are based on rates for MD degrees from US institutions.

KPI-15: National Academy Memberships: National Academy Memberships held by faculty as reported by the Center for Measuring University Performance in the Top American Research Universities (TARU) annual report or the official membership directories maintained by each national academy.

KPI-16: Faculty Awards: Awards include: American Council of Learned Societies (ACLS) Fellows, Beckman Young Investigators, Burroughs Wellcome Fund Career Awards, Cottrell Scholars, Fulbright American Scholars, Getty Scholars in Residence, Guggenheim Fellows, Howard Hughes Medical Institute Investigators, Lasker Medical Research Awards, MacArthur Foundation Fellows, Andrew W. Mellon Foundation Distinguished Achievement Awards, National Endowment for the Humanities (NEH) Fellows, National Humanities Center Fellows, National Institutes of Health (NIH) MERIT, National Medal of Science and National Medal of Technology, NSF CAREER awards (excluding those who are also PECASE winners), Newberry Library Long-term Fellows, Pew Scholars in Biomedicine, Presidential Early Career Awards for Scientists and Engineers (PECASE), Robert Wood Johnson Policy Fellows, Searle Scholars, Sloan Research Fellows, Woodrow Wilson Fellows. 


\section{DEFINITIONS (cont.)}

KPI-17: Total Research Expenditures: Total expenditures (in millions of dollars) for all research activities (including non-science and engineering activities). Source: As reported by each institution to the National Science Foundation annual survey of Higher Education Research and Development (HERD) based on the NSF rules and definitions.

KPI-18: Research Expenditures Funded from External Sources: This metric reports the amount of research expenditures that was funded from federal, private industry and other (non-state and non-institutional) sources. Source: As reported by each institution to the National Science Foundation annual survey of Higher Education Research and Development (HERD) based on the NSF rules and definitions.

KPI-19: Utility Patents Awarded [from the USPTO]: The number of utility patents awarded by the United States Patent and Trademark Office (USPTO) by Calendar year - does not include design, plant or other types.

KPI-20: Number of Licenses/Options Executed Annually: Licenses/options executed in the fiscal year for all technologies - as reported by universities on the Association of University Technology Managers Annual (AUTM) annual Licensing Survey.

KPI-21: Number of Start-up Companies Created: The number of start-up companies that were dependent upon the licensing of University technology for initiation - as reported by universities on the Association of University Technology Managers Annual (AUTM) annual Licensing Survey.

\section{Enrollment Planning (ENRL)}

ENRL-1: Fall Headcount Enrollment by Student Level and Student Type: This table reports the number of students enrolled by student type categories. These headcounts only include those students who were seeking a degree unclassified students (eg, dual enrolled) are not included. The student type for undergraduates is based on the 'Type of Student at Most Recent Admission'. The First Time in College (FTIC) student was admitted in the same fall term or in the preceding summer term - this includes those who were re-admitted as FTICs.

ENRL-2: Percent of Resident Baccalaureate-Seeking Resident Undergraduates Earning 15+ Credits: This table reports the percent of baccalaureate-seeking resident undergraduates who earned fifteen or more credit hours during the fall term as reported on the Term Credit Hours Earned element (\#01089). This includes the pass/fail courses in which the student earned a passing grade and excludes audited courses.

ENRL-3 Full-Time Equivalent Enrollment by Course Level: This table reports Full-time Equivalent (FTE) enrollment which is a measure of all instructional activity (regardless of fundability) that is based on the number of credit hours that students enroll. This FTE calculation is based on the standard national definition, which divides undergraduate credit hours by 30 and graduate credit hours by 24. Pursuant to section 1013.31, Florida Statutes, Board facilities staff use this data as a key factor in the calculation of facility space needs for institution educational plant surveys.

ENRL-4: Percent FTE Enrollment by Method of Instruction: This table reports the percentages of FTE enrollment that is classified as Distance Learning for all students at all campuses regardless of funding source. Distance Learning is a course in which at least 80 percent of the direct instruction of the course is delivered using some form of technology when the student and instructor are separated by time or space, or both (per 1009.24(17), F.S.). 


\section{$\sigma$ \\ STATE UNIVERSITY SYSTEM OF FLORIDA}

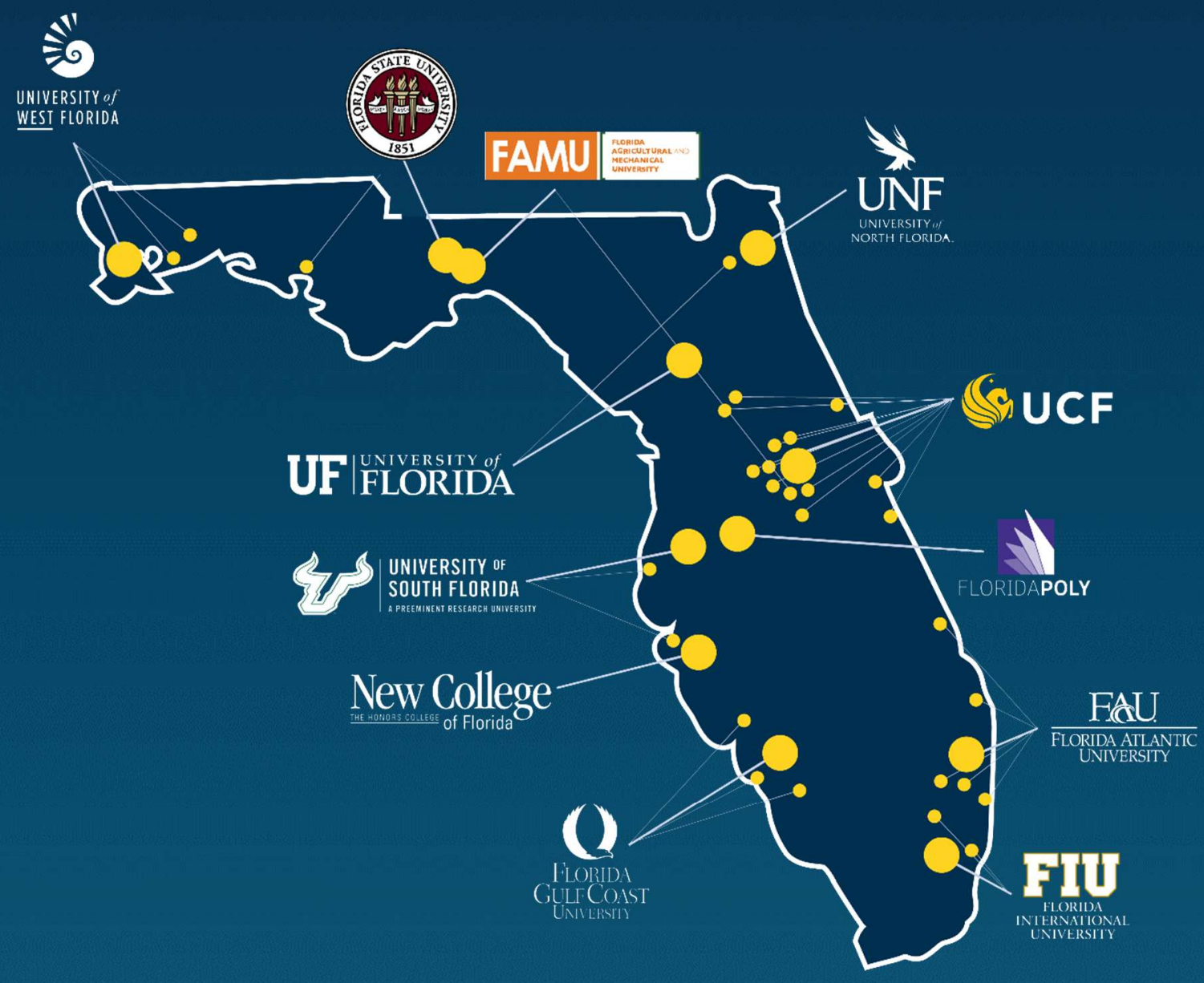

